\title{
Comultiplication in link Floer homology and transversely nonsimple links
}

\author{
JOHN A BALDWIN
}

For a word $w$ in the braid group $B_{n}$, we denote by $T_{w}$ the corresponding transverse braid in $\left(\mathbb{R}^{3}, \xi_{\text {rot }}\right)$. We exhibit, for any two $g, h \in B_{n}$, a "comultiplication" map on link Floer homology $\widetilde{\Phi}: \widetilde{H F L}\left(m\left(T_{h g}\right)\right) \rightarrow \widetilde{H F L}\left(m\left(T_{g} \# T_{h}\right)\right)$ which sends $\tilde{\theta}\left(T_{h g}\right)$ to $\widetilde{\theta}\left(T_{g} \# T_{h}\right)$. We use this comultiplication map to generate infinitely many new examples of prime topological link types which are not transversely simple.

57M27, 57R 17

\section{Introduction}

Transverse links feature prominently in the study of contact 3-manifolds. They arise very naturally - for instance, as binding components of open book decompositions - and can be used to discern important properties of the contact structures in which they sit (see Baker, Etnyre and Van Horn-Morris [1, Theorem 1.15] for a recent example). Yet, transverse links, even in the standard tight contact structure $\xi_{\text {std }}$ on $\mathbb{R}^{3}$, are notoriously difficult to classify up to transverse isotopy.

A transverse link $T$ comes equipped with two "classical" invariants which are preserved under transverse isotopy: its topological link type and its self-linking number $\operatorname{sl}(T)$. For transverse links with more than one component, it makes sense to refine the notion of self-linking number as follows. Let $T$ and $T^{\prime}$ be two transverse representatives of some $l$-component topological link type, and suppose there are labelings $T=T_{1} \cup \cdots \cup T_{l}$ and $T^{\prime}=T_{1}^{\prime} \cup \cdots \cup T_{l}^{\prime}$ of the components of $T$ and $T^{\prime}$ such that

(1) there is a topological isotopy sending $T$ to $T^{\prime}$ which sends $T_{i}$ to $T_{i}^{\prime}$ for each $i$,

(2) $\operatorname{sl}(S)=\operatorname{sl}\left(S^{\prime}\right)$ for any sublinks $S=T_{n_{1}} \cup \cdots \cup T_{n_{j}}$ and $S^{\prime}=T_{n_{1}}^{\prime} \cup \cdots \cup T_{n_{j}}^{\prime}$.

Then we say that $T$ and $T^{\prime}$ have the same self-linking data, and we write $\mathcal{S} \mathcal{L}(T)=$ $\mathcal{S} \mathcal{L}\left(T^{\prime}\right)$. A basic question in contact geometry is how to tell, given two transverse representatives $T$ and $T^{\prime}$ of some topological link with the same self-linking data, whether $T$ and $T^{\prime}$ are transversely isotopic; that is, whether the classical data completely determines the transverse link type. We say that a topological link type is transversely simple if any two transverse representatives $T$ and $T^{\prime}$ which satisfy $\mathcal{S} \mathcal{L}(T)=\mathcal{S} \mathcal{L}\left(T^{\prime}\right)$ are transversely isotopic. Otherwise, the link type is said to be transversely nonsimple. 
From this point on, we shall restrict our attention to transverse links in the tight rotationally symmetric contact structure $\xi_{\text {rot }}$ on $\mathbb{R}^{3}$, which is contactomorphic to $\xi_{\text {std }}$. There are several well-known examples of knot types which are transversely simple. Among these are the unknot by Eliashberg [7], torus knots by Etnyre [9] and the figure eight by Etnyre and Honda [10].

Only recently, however, have knot types been discovered which are not transversely simple. These include a family of 3-braids found by Birman and Menasco [5] using the theory of braid foliations and the $(2,3)$ cable of the $(2,3)$ torus knot, which was shown to be transversely nonsimple by Etnyre and Honda using contact-geometric techniques [12]. Matsuda and Menasco have since identified two explicit transverse representatives of this cabled torus knot which have identical self-linking numbers, but which are not transversely isotopic [18]. Their examples take center stage in Section 5 of this paper.

There has been a flurry of progress in finding transversely nonsimple link types in the last couple years, spurred by the discovery of a transverse invariant $\theta$ in link Floer homology by Ozsváth, Szabó and Thurston [25]; this discovery, in turn, was made possible by the combinatorial description of $H F L^{-}$found by Manolescu, Ozsvath and Sarkar in [16] (see also Manolescu, Ozsváth, Szabó and Thurston [17]). ${ }^{1}$ This $\theta$ invariant is applied by $\mathrm{Ng}$, Ozsváth and Thurston in [20] to identify several examples of transversely nonsimple links, including the knot 10132. In [28], Vértesi proves a connected sum formula for $\theta$, which she wields to find infinitely many examples of nonprime knots which are transversely nonsimple (Kawamura has since proven a similar result without using Floer homology [13]; both hers and Vértesi's results follow from Etnyre and Honda's work on Legendrian connected sums [11]).

Finding infinite families of transversely nonsimple prime knots is generally more difficult. Using a slightly different invariant, which we shall denote by $\underline{\theta}$, derived from knot Floer homology and discovered by Lisca, Ozsváth, Szabó and Stipsicz in [15], Ozsváth and Stipsicz identify such an infinite family among two-bridge knots [23]. And, most recently, Khandhawit and $\mathrm{Ng}$ use the invariant $\theta$ to construct a 2-parameter infinite family of prime transversely nonsimple knots, which generalizes the example of $10_{132}$ [14].

In this paper, we formulate and apply a strategy for generating a slew of new infinite families of transversely nonsimple prime links. This strategy hinges on the "naturality" results below. For a word $w$ in the braid group $B_{n}$, we denote by $T_{w}$ the corresponding transverse braid in $\left(\mathbb{R}^{3}, \xi_{\text {rot }}\right)$.

${ }^{1}$ There are several versions of this $\theta$ invariant, denoted by $\theta^{-}, \hat{\theta}$ and $\tilde{\theta}$. 


\section{Theorem 1.1 There exists a map on link Floer homology}

$$
\widetilde{\Phi}: \widetilde{H F L}\left(m\left(T_{w \sigma_{i}}\right)\right) \rightarrow \widetilde{H F L}\left(m\left(T_{w}\right)\right)
$$

which sends $\tilde{\theta}\left(T_{w \sigma_{i}}\right)$ to $\tilde{\theta}\left(T_{w}\right)$, where $\sigma_{i}$ is one of the standard generators of $B_{n}$.

This theorem implies the existence of a "comultiplication" map on link Floer homology, similar in spirit to the map which we discovered in [2]:

Theorem 1.2 For any two braid words $h$ and $g$ in $B_{n}$, there exists a map

$$
\tilde{\mu}: \widetilde{H F L}\left(m\left(T_{h g}\right)\right) \rightarrow \widetilde{H F L}\left(m\left(T_{g} \# T_{h}\right)\right)
$$

which sends $\tilde{\theta}\left(T_{h g}\right)$ to $\tilde{\theta}\left(T_{g} \# T_{h}\right)$.

One may combine Theorem 1.2 with Vértesi's result governing the behavior of $\theta$ under connected sums to conclude the following.

Theorem 1.3 If $\hat{\theta}\left(T_{g}\right)$ and $\hat{\theta}\left(T_{h}\right)$ are both nonzero, then so is $\hat{\theta}\left(T_{h g}\right)$.

Here, we sketch one potential way to use these results to find transversely nonsimple links. Start with some $w_{1}, w_{2} \in B_{n}$ for which $T_{w_{1}}$ and $T_{w_{2}}$ are topologically isotopic and have the same self-linking data, but for which $\hat{\theta}\left(T_{w_{1}}\right)=0$ while $\hat{\theta}\left(T_{w_{2}}\right) \neq 0$, so that $T_{w_{1}}$ and $T_{w_{2}}$ are not transversely isotopic. Now, choose an $h \in B_{n}$ for which $\hat{\theta}\left(T_{h}\right) \neq 0$. Theorem 1.3 then implies that $\hat{\theta}\left(T_{h w_{2}}\right) \neq 0$ as well. If one can show that $\hat{\theta}\left(T_{h w_{1}}\right)=0$, that $T_{h w_{1}}$ and $T_{h w_{2}}$ still represent the same topological link type, and that $\mathcal{S} \mathcal{L}\left(T_{h w_{1}}\right)=\mathcal{S} \mathcal{L}\left(T_{h w_{2}}\right)$ (this is automatic if $T_{h w_{1}}$ and $T_{h w_{2}}$ are knots), then one may conclude that $T_{h w_{1}}$ represents a transversely nonsimple link type.

An advantage of this approach for generating new transversely nonsimple link types from old over, say, that of Vértesi [28] and Kawamuro [13], is that there is no a priori reason to expect that the links so formed are composite. We demonstrate the effectiveness of this approach in Section 5 of this paper. In doing so, we describe an infinite family of prime transversely nonsimple link types (half are knots; the other half are 3-component links) which generalizes the $(2,3)$ cable of the $(2,3)$ torus knot. Moreover, it is clear that this example only scratches the surface of the potential of our more general technique.

Lastly, it is tempting to conjecture that the two invariants $\theta$ and $\underline{\theta}$ agree for transverse links in $\left(\mathbb{R}^{3}, \xi_{\text {rot }}\right)$, as they share many formal properties. We prove a partial result in this direction, which follows from Theorem 1.1 together with work of Vela-Vick on the $\underline{\theta}$ invariant [27].

Theorem $1.4 \hat{\theta}(T)$ and $\underline{\hat{\theta}}(T)$ agree for positive, transverse, connected braids $T$ in $\left(\mathbb{R}^{3}, \xi_{\text {rot }}\right)$. 


\section{Organization}

In the next section, we outline the relationship between grid diagrams, Legendrian links and their transverse pushoffs. In Section 3, we review the grid diagram construction of link Floer homology and describe some important properties of the transverse invariant $\theta$. In Section 4, we prove Theorems 1.1, 1.2, 1.3 and 1.4. And, in Section 5, we outline a general strategy for using our comultiplication result to produce new examples of transversely nonsimple link types, and we give an infinite family of such examples which are prime.

Acknowledgements I wish to thank Lenny $\mathrm{Ng}$ for helpful correspondence. His suggestions were key in developing some of the strategy formulated in Section 5. Thanks also to the referee for helpful comments.

The author was supported by an NSF Postdoctoral Fellowship.

\section{Grid diagrams, Legendrian and transverse links}

In this section, we provide a brief review of the relationship between Legendrian links in $\left(\mathbb{R}^{3}, \xi_{\text {std }}\right)$, transverse braids in $\left(\mathbb{R}^{3}, \xi_{\text {rot }}\right)$ and grid diagrams, largely following the discussion in [14]. For a more detailed account, see Khandhawit and $\mathrm{Ng}$ [14] and $\mathrm{Ng}$ and Thurston [21]. The standard tight contact structure $\xi_{\text {std }}$ on $\mathbb{R}^{3}$ is given as

$$
\xi_{\text {std }}=\operatorname{ker}(d z-y d x) .
$$

An oriented link $L \subset\left(\mathbb{R}^{3}, \xi_{\text {std }}\right)$ is called Legendrian if it is everywhere tangent to $\xi_{\text {std }}$, and transverse if it is everywhere transverse to $\xi_{\text {std }}$ such that $d z-y d x>0$ along the orientation of $L$. Any smooth link can be perturbed by a $C^{0}$ isotopy to be Legendrian or transverse. We say that two Legendrian (resp. transverse) links are Legendrian (resp. transversely) isotopic if they are isotopic through Legendrian (resp. transverse) links.

A Legendrian link $L$ can be perturbed to a transverse link (which is arbitrarily close to $L$ in the $C^{\infty}$ topology) by pushing $L$ along its length in a generic direction transverse to the contact planes in such a way that the orientation of the pushoff agrees with that of $L$. The resulting link $L^{+}$is called a positive transverse pushoff of $L$. Legendrian isotopic links give rise to transversely isotopic pushoffs. Conversely, every transverse link is the positive transverse pushoff of some Legendrian link; however, two such Legendrian links need not be Legendrian isotopic. The precise relationship between Legendrian and transverse links is best explained via front projections.

The front projection of a Legendrian link is its projection onto the $x z$ plane. The front projection of a generic Legendrian link has no vertical tangencies and has only 
semicubical cusps and transverse double points as singularities. Moreover, at each double point, the slope of the overcrossing is more negative than the slope of the undercrossing. See Figure 2 (c) for the front projection of a right-handed Legendrian trefoil.

The positive (resp. negative) stabilization of a Legendrian link $L$ along some component $C$ of $L$ is the Legendrian link whose front projection is obtained from that of $L$ by adding a zigzag along $C$ with downward (resp. upward) pointing cusps. See Figure 1. Two Legendrian links are said to be negatively stably isotopic if they are Legendrian isotopic after each has been negatively stabilized some number of times along some of its components. The following theorem implies that the classification of transverse links up to transverse isotopy is equivalent to the classification of Legendrian links up to Legendrian isotopy and negative stabilization.

(a)

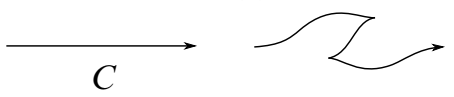

(b)

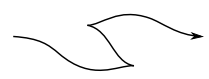

Figure 1: (a) and (b) are local pictures of the positive and negative stabilizations, respectively, of a Legendrian link along one of its components $C$.

Theorem 2.1 [8; 22] Two Legendrian links are negatively stably isotopic if and only if their positive transverse pushoffs are transversely isotopic.

Consider the rotationally symmetric tight contact structure on $\mathbb{R}^{3}$ defined by

$$
\xi_{\text {rot }}=\operatorname{ker}(d z-y d x+x d y) .
$$

The diffeomorphism of $\mathbb{R}^{3}$ given by

$$
\phi(x, y, z)=(x, 2 y, x y+z)
$$

sends $\xi_{\text {rot }}$ to $\xi_{\text {std }}$. One can define transverse links for $\xi_{\text {rot }}$ in the same way that one does for $\xi_{\text {std }}$. Since $\phi$ sends a transverse link in $\left(\mathbb{R}^{3}, \xi_{\text {rot }}\right)$ to a transverse link in $\left(\mathbb{R}^{3}, \xi_{\text {std }}\right)$, the study of transverse links in $\left(\mathbb{R}^{3}, \xi_{\text {std }}\right)$ is equivalent to that in $\left(\mathbb{R}^{3}, \xi_{\text {rot }}\right)$; however, the latter is often more convenient, per the following theorem of Bennequin.

Theorem 2.2 [3] Any transverse link in $\left(\mathbb{R}^{3}, \xi_{\text {rot }}\right)$ is transversely isotopic to a closed braid around the $z$-axis.

Theorem 2.2 allows us to use braid-theoretic techniques to study transverse links. For a braid word $w \in B_{n}$, we let $T_{w}$ denote the corresponding transverse braid around 
the $z$-axis. Braid words which are conjugate in $B_{n}$ clearly correspond to transversely isotopic links. Recall that, for $w \in B_{n}$, a positive (resp. negative) braid stabilization of $w$ is the operation which replaces $w$ by the word $w \sigma_{n}$ (resp. $w \sigma_{n}^{-1}$ ) in $B_{n+1}$. We will also refer to $T_{w \sigma_{n}}$ (resp. $T_{w \sigma_{n}^{-1}}$ ) as the positive (resp. negative) braid stabilization of the transverse link $T_{w}$. The following theorem makes precise the relationship between braids and transverse links in $\left(\mathbb{R}^{3}, \xi_{\text {rot }}\right)$.

Theorem $2.3[22 ; 30] \quad$ For $w \in B_{n}$ and $w^{\prime} \in B_{m}$, the transverse links $T_{w}$ and $T_{w^{\prime}}$ are transversely isotopic in $\left(\mathbb{R}^{3}, \xi_{\text {rot }}\right)$ if and only if $w$ and $w^{\prime}$ are related by a sequence of conjugations and positive braid stabilizations and destabilizations.

In Section 5, we use a braid operation called an exchange move. If $a, b$ and $c$ in $B_{n}$ are words in the generators $\sigma_{2}, \ldots, \sigma_{n-1}$, then an exchange move is the operation which replaces the word $w_{1}=a \sigma_{1} b \sigma_{1}^{-1} c$ with the word $w_{2}=a \sigma_{1}^{-1} b \sigma_{1} c$. An exchange move is actually just a composition of conjugations, one positive braid stabilization and one positive destabilization, and so the link $T_{w_{1}}$ is transversely isotopic to $T_{w_{2}}$ (see, for example, $\mathrm{Ng}$ and Thurston [21]).

It bears mentioning that the self-linking number of a transverse link admits a nice formulation in the language of braids. If $\Sigma$ is a Seifert surface for a transverse link $T$, then the vector bundle $\xi_{\text {rot }} \mid \Sigma$ is trivial and, therefore, has a nonzero section $v$. Recall that the self-linking number of $T$ is defined by

$$
\operatorname{sl}(T)=\operatorname{lk}\left(T, T^{\prime}\right),
$$

where $T^{\prime}$ is a pushoff of $T$ in the direction of $v$. Any two links which are transversely isotopic have identical self-linking numbers. For a word $w \in B_{n}$, the self-linking number of $T_{w}$ is given simply by $a(w)-n$, where $a(w)$ is the algebraic length of $w$.

In what remains of this section, we describe a relationship between the front diagram of a Legendrian link in $\left(\mathbb{R}^{3}, \xi_{\text {std }}\right)$ and a braid representation of its positive transverse pushoff, thought of as a transverse link in $\left(\mathbb{R}^{3}, \xi_{\text {rot }}\right)$. Grid diagrams provide the necessary connection.

A grid diagram $G$ is an $k \times k$ square grid along with a collection of $k X$ 's and $k O$ 's contained in these squares such that every row and column contains exactly one $O$ and one $X$ and no square contains both an $O$ and an $X$. See Figure 2 (a). We call $k$ the grid number of $G$. One can produce an oriented link diagram $L$ from $G$ by drawing a horizontal segment from the $O$ 's to the $X$ 's in each row and a vertical segment from the $X$ 's to the $O$ 's in each column so that the horizontal segments pass over the vertical segments (this is the convention used by Khandhawit and $\mathrm{Ng}$ [14], and the opposite of the convention in Manolescu, Ozsváth and Sarkar [16]; see Ng and 
Thurston [21] for a discussion on the relationship between the two conventions), as in Figure 2 (b). By rotating $L 45^{\circ}$ clockwise, and then smoothing the upward and downward pointing corners and turning the leftward and rightward pointing corners into cusps, one obtains the front projection of a Legendrian link, as in Figure 2 (c). Let us denote this Legendrian link by $L(G)$.

(a)

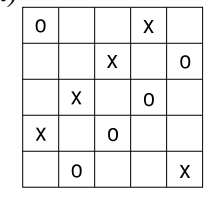

(b)

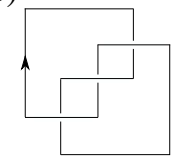

(d)

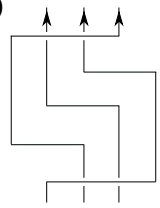

(c)

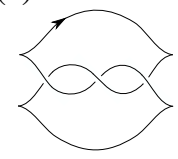

(e)

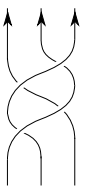

Figure 2: In (a), a grid diagram $G$. In (b), the oriented link corresponding to $G$. In (c), a front projection for the Legendrian link $L(G)$. In (d) and (e), the braid corresponding to $G$. Here, $w(G)=\sigma_{1} \sigma_{2} \sigma_{1} \sigma_{2}$.

Alternatively, one can construct a braid diagram from $G$ by drawing a horizontal segment from the $O$ 's to the $X$ 's in each row, as before, and drawing a vertical segment from the $X$ 's to the $O$ 's for each column in which the marking $X$ lies under the marking $O$. For those columns in which the $X$ is above the $O$, we draw two vertical segments: one from the $X$ up to the top of the grid diagram, and the other from the bottom of the grid diagram up to the $O$. As before, we require that the horizontal segments pass over the vertical segments. Note that all vertical segments are oriented upwards and that the closure of the diagram we have constructed is a braid. See Figures 2 (d) and 2 (e) for an example of this procedure. Let us denote the corresponding braid word by $w(G)$, read from the bottom up. The relationship between $T_{w(G)}$ and $(L(G))^{+}$is expressed in the proposition below.

Proposition 2.4 [14, Proposition 3] The contactomorphism $\phi$ from $\left(\mathbb{R}^{3}, \xi_{\text {rot }}\right)$ to $\left(\mathbb{R}^{3}, \xi_{\text {std }}\right)$ defined in Equation (1) sends the transverse link $T_{w(G)}$ to a link which is transversely isotopic to $(L(G))^{+}$.

\section{Link Floer homology and the transverse invariant}

In this section, we describe the grid diagram formulation of link Floer homology discovered in $[16 ; 17]$. Let $G$ be a grid diagram for a link $L$ and suppose that $G$ has 
grid number $k$. From this point forward, we think of $G$ as a toroidal grid diagram that is, we identify the top and bottom sides of $G$ and the right and left sides of $G$ - so that the horizontal and vertical lines become $k$ horizontal and $k$ vertical circles. Let (I) and $\mathbb{X}$ denote the sets of markings $\left\{O_{i}\right\}_{i=1}^{k}$ and $\left\{X_{i}\right\}_{i=1}^{k}$, respectively.

We associate to $G$ a chain complex $\left(\mathrm{CFL}^{-}(m(L)), \partial^{-}\right)$as follows. The generators of $C_{F L}(L)$ are one-to-one correspondences between the horizontal and vertical circles of $G$. Equivalently, we may think of a generator as a set of $k$ intersection points between the horizontal and vertical circles, such that no intersection point appears on more than one horizontal circle or on more than one vertical circle. We denote this set of generators by $\mathbf{S}(G)$. Then, $C F L^{-}(m(L))$ is defined to be the free $\mathbb{Z}_{2}\left[U_{1}, \ldots, U_{k}\right]$-module generated by the elements of $\mathbf{S}(G)$, where the $U_{i}$ are formal variables corresponding to the markings $O_{i}$.

For $\mathbf{x}, \mathbf{y} \in \mathbf{S}(G)$, we let $\operatorname{Rect}_{G}(\mathbf{x}, \mathbf{y})$ denote the space of embedded rectangles in $G$ with the following properties. $\operatorname{Rect}_{G}(\mathbf{x}, \mathbf{y})$ is empty unless $\mathbf{x}$ and $\mathbf{y}$ coincide at $k-2$ points. An element $r \in \operatorname{Rect}_{G}(\mathbf{x}, \mathbf{y})$ is an embedded disk on the toroidal grid $G$ whose edges are arcs on the horizontal and vertical circles and whose four corners are intersection points in $\mathbf{x} \cup \mathbf{y}$. Moreover, we stipulate that if we traverse each horizontal boundary component of $r$ in the direction specified by the induced orientation on $\partial r$, then this horizontal arc is oriented from a point in $\mathbf{x}$ to a point in $\mathbf{y}$. If $\operatorname{Rect}_{G}(\mathbf{x}, \mathbf{y})$ is nonempty, then it consists of exactly two rectangles. See Figure 3 for an example. We let $\operatorname{Rect}_{G}^{o}(\mathbf{x}, \mathbf{y})$ denote the space of $r \in \operatorname{Rect}_{G}^{o}(\mathbf{x}, \mathbf{y})$ for which $r \cap \mathbb{X}=\operatorname{Int}(r) \cap \mathbf{x}=\varnothing$.

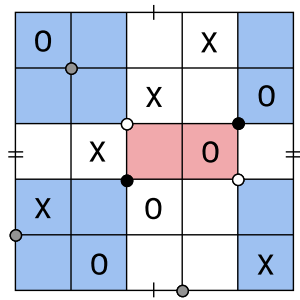

Figure 3: A grid diagram $G$ for the right-handed trefoil. The generator $\mathbf{x}$ comprises the black and gray intersection points while $\mathbf{y}$ comprises the white and gray intersection points. $\operatorname{Rect}_{G}(\mathbf{x}, \mathbf{y})$ contains the shaded rectangles in red and blue, while $\operatorname{Rect}_{G}^{o}(\mathbf{x}, \mathbf{y})$ contains only the red rectangle.

The module $\mathrm{CFL}^{-}(m(L))$ is endowed with an endomorphism

$$
\partial^{-}: C F L^{-}(m(L)) \rightarrow C F L^{-}(m(L)),
$$


defined on $\mathbf{S}(G)$ by

$$
\partial^{-}(\mathbf{x})=\sum_{\mathbf{y} \in \mathbf{S}(G)} \sum_{r \in \operatorname{Rect}_{G}^{o}(\mathbf{x}, \mathbf{y})} U_{1}^{O_{1}(r)} \cdots U_{k}^{O_{n}(r)} \cdot \mathbf{y} .
$$

Here, $O_{i}(r)$ denotes the number of times the marking $O_{i}$ appears in $r$. The map $\partial^{-}$is a differential, and, so, gives rise to a chain complex $\left(C F L^{-}(m(L)), \partial^{-}\right)$. The homology of this chain complex, $H F L^{-}(m(L))=H_{*}\left(C F L^{-}(m(L)), \partial^{-}\right)$, is an invariant of the link $L$, and agrees with the link Floer homology of $m(L)$ defined in [24]. It bears mentioning that the complex $\left(C F L^{-}\left(m(L), \partial^{-}\right)\right.$comes equipped with Maslov and Alexander gradings, which are then inherited by $\mathrm{HFL}^{-}(m(L))$; however, we will not discuss these gradings further as they play no role in this paper.

Suppose that the link $L$ has $l$ components. If $O_{i}$ and $O_{j}$ lie on the same component of $L$, then multiplication by $U_{i}$ in $\left(C F L^{-}(m(L)), \partial^{-}\right)$is chain homotopic to multiplication by $U_{j}$, and, so, these multiplications induce the same maps on $\mathrm{HFL}^{-}(m(L))$ [17, Lemma 2.9]. So, if we label the markings in $\mathbb{O}$ so that $O_{1}, \ldots, O_{l}$ lie on different components, then we can think of $H F L^{-}(m(L))$ as a module over $\mathbb{Z}_{2}\left[U_{1}, \ldots, U_{l}\right]$.

Setting $U_{1}=\cdots=U_{l}=0$, one obtains a chain complex $(\widehat{C F L}(m(L)), \widehat{\partial})$ whose homology we denote by $\widehat{H F L}(m(L))$. The latter is a bigraded vector space over $\mathbb{Z}_{2}$, whose graded Euler characteristic is some normalization of the multivariable Alexander polynomial of $m(L)$ [24]. If one sets $U_{1}=\cdots=U_{k}=0$, one obtains a chain complex $(\widetilde{C F L}(m(L)), \widetilde{\partial})$ whose homology we denote by $\widetilde{H F L}(m(L))$. The group $\widehat{H F L}(m(L))$ determines $\widetilde{H F L}(m(L))$. Specifically, if we let $n_{i}$, for $i=1, \ldots, l$, denote the number of markings in $\mathbb{O}$ on the $i$-th component of $L$, then

$$
\widetilde{H F L}(m(L))=\widehat{H F L}(m(L)) \otimes \bigotimes_{i=1}^{l} V_{i}^{\otimes\left(n_{i}-1\right)},
$$

where $V_{i}$ is a fixed two dimensional vector space [17, Proposition 2.13], and the quotient map

$$
j: \widehat{C F L}(m(L)) \rightarrow \widetilde{C F L}(m(L))
$$

induces an injection $j_{*}$ on homology.

The element $\mathbf{z}^{+}(G) \in \mathbf{S}(G)$, which consists of the intersection points at the upper right corners of the squares in $G$ containing the markings in $\mathbb{X}$, is clearly a cycle in $\left(C F L^{-}(m(L)), \partial^{-}\right)$(and, hence, also in the other chain complexes). If $T$ is the transverse link in $\left(\mathbb{R}^{3}, \xi_{\text {rot }}\right)$ corresponding to the braid obtained from $G$ as in Figure 2 (e), then $T$ is topologically isotopic to $L$, and the image of $\mathbf{z}^{+}(G)$ in $H F L^{-}(m(T))$ is the transverse invariant $\theta^{-}(T)$ defined in [25]. The images of $\mathbf{z}^{+}(G)$ in $\widehat{H F L}(m(T))$ and $\widetilde{H F L}(m(T))$ are likewise denoted $\hat{\theta}(T)$ and $\widetilde{\theta}(T)$, and are invariants of the transverse 
link $T$ as well. Moreover, the map $j_{*}$ sends $\hat{\theta}(T)$ to $\tilde{\theta}(T)$; in particular, $\hat{\theta}(T)=0$ if and only if $\tilde{\theta}(T)=0$. The theorem below makes these statements about invariance precise.

Theorem 3.1 [25, Theorem 7.1] Suppose that $G$ and $G^{\prime}$ are two grid diagrams whose associated braids $T$ and $T^{\prime}$ are transversely isotopic. Then, there is an isomorphism

$$
f_{*}^{o}: \operatorname{HFL}^{o}(m(T)) \rightarrow \operatorname{HFL}^{o}\left(m\left(T^{\prime}\right)\right),
$$

induced by a chain map $f^{o}$, which sends $\theta^{o}(T)$ to $\theta^{o}\left(T^{\prime}\right)$.

Here, the superscript " $o$ " is meant to indicate that this theorem holds for any of the three versions of link Floer homology described above. In particular, if $T$ and $T^{\prime}$ are two transverse links for which $\hat{\theta}(T) \neq 0$ and $\hat{\theta}\left(T^{\prime}\right)=0$, then $T$ and $T^{\prime}$ are not transversely isotopic (the invariant $\theta^{-}(T)$ is always nonzero and non- $U_{i}$-torsion in $H F L^{-}(m(T))$ [25, Theorem 7.3]). These transverse invariants also behave nicely under negative braid stabilizations.

Theorem 3.2 [25, Theorem 7.2] Suppose that $G$ and $G^{\prime}$ are two grid diagrams with associated braids $T$ and $T^{\prime}$, and suppose that $T^{\prime}$ is obtained from $T$ by performing a negative braid stabilization along the $i$-th component of $T$. Then, there is an isomorphism

$$
f_{*}^{-}: H F L^{-}(m(T)) \rightarrow H F L^{-}\left(m\left(T^{\prime}\right)\right),
$$

induced by a chain map $f^{-}$, which sends $\theta(T)$ to $U_{i} \cdot \theta\left(T^{\prime}\right)$.

Since multiplication by $U_{i}$ is the same as multiplication by zero on $\widehat{H F L}$ and $\widetilde{H F L}$, we obtain the following corollary.

Corollary 3.3 If $T^{\prime}$ is obtained from a transverse braid $T$ by performing a negative braid stabilization along some component of $T$, then $\hat{\theta}\left(T^{\prime}\right)=\widetilde{\theta}\left(T^{\prime}\right)=0$.

\section{The map $\Phi$ and comultiplication}

Fix some $w \in B_{n}$ and some $i \in\{1, \ldots, n-1\}$. Figure 4 shows simultaneously a portion of a grid diagram $G_{\beta}$ for $T_{w \sigma_{i}}$ and the corresponding portion of a grid diagram $G_{\gamma}$ for $T_{w}$. The grid diagrams $G_{\beta}$ and $G_{\gamma}$ are the same except that $G_{\beta}$ uses the horizontal curve $\beta$ while $G_{\gamma}$ uses the horizontal curve $\gamma$. Let $k$ denote their common grid number. 


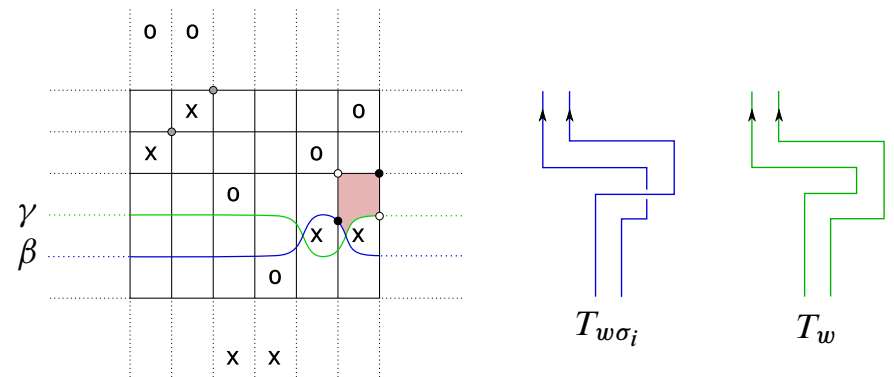

Figure 4: A portion of the grid diagrams $G_{\beta}$ and $G_{\gamma}$. The cycle $\mathbf{z}^{+}\left(G_{\beta}\right)$ is shown as a collection of black and gray dots, while $\mathbf{z}^{+}\left(G_{\gamma}\right)$ is represented by the white and gray dots.

For $\mathbf{x} \in \mathbf{S}\left(G_{\beta}\right)$ and $\mathbf{y} \in \mathbf{S}\left(G_{\gamma}\right)$, let Pent $\operatorname{Pr}_{\beta \gamma}(\mathbf{x}, \mathbf{y})$ denote the space of embedded pentagons with the following properties. Pent ${ }_{\beta \gamma}(\mathbf{x}, \mathbf{y})$ is empty unless $\mathbf{x}$ and $\mathbf{y}$ coincide at $k-2$ points. An element $p \in \operatorname{Pent}_{\beta \gamma}(\mathbf{x}, \mathbf{y})$ is an embedded disk in the torus whose boundary consists of five arcs, each contained in horizontal or vertical circles. We stipulate that under the orientation induced on the boundary of $p$, the boundary may be traversed as follows. Start at the component of $\mathbf{x}$ on the curve $\beta$ and proceed along an arc contained in $\beta$ until we arrive at the right-most intersection point between $\beta$ and $\gamma$; next, proceed along an arc contained in $\gamma$ until we reach the component of $\mathbf{y}$ contained in $\gamma$; next, follow the arc of a vertical circle until we arrive at a component of $\mathbf{x}$; then, proceed along the arc of a horizontal circle until we arrive at a component of $\mathbf{y}$; finally, follow an arc contained in a vertical circle back to the initial component of $\mathbf{x}$. Let Pent $_{\beta \gamma}^{o}(\mathbf{x}, \mathbf{y})$ denote the space of $p \in \operatorname{Pent}_{\beta \gamma}(\mathbf{x}, \mathbf{y})$ for which $p \cap \mathbb{X}=\operatorname{Int}(p) \cap \mathbf{x}=\varnothing$. We construct a map

$$
\phi^{-}: C F L^{-}\left(m\left(T_{w \sigma_{i}}\right)\right) \rightarrow C F L^{-}\left(m\left(T_{w}\right)\right)
$$

of $\mathbb{Z}_{2}\left[U_{1}, \ldots, U_{k}\right]$-modules as follows. For $\mathbf{x} \in \mathbf{S}\left(G_{\beta}\right)$, let

We then define

$$
\phi^{-}(\mathbf{x})=\sum_{\mathbf{y} \in \mathbf{S}\left(G_{\gamma}\right)} \sum_{p \in \operatorname{Pent}_{\beta \gamma}^{o}(\mathbf{x}, \mathbf{y})} U_{1}^{O_{1}(p)} \cdots U_{k}^{O_{n}(p)} \cdot \mathbf{y} .
$$

$$
\widetilde{\phi}: \widetilde{C F L}\left(m\left(T_{w \sigma_{i}}\right)\right) \rightarrow \widetilde{C F L}\left(m\left(T_{w}\right)\right)
$$

to be the map on $\widetilde{C F L}$ induced by $\phi^{-}$. In other words, $\tilde{\phi}$ counts pentagons in Pent $_{\beta \gamma}^{o}(\mathbf{x}, \mathbf{y})$ which also miss the $\mathbb{O}$ basepoints. (This construction is inspired by the proof of commutation invariance in [17, Section 3.1].)

$\operatorname{Remark}$ 4.1 Unlike $\widetilde{\phi}$, the map $\phi^{-}$is not necessarily a chain map. 
The juxtaposition $p * r$ of any $p \in \operatorname{Pent}_{\beta \gamma}^{o}(\mathbf{x}, \mathbf{y})$ with any rectangle $r \in \operatorname{Rect}_{G_{\gamma}}^{o}(\mathbf{y}, \mathbf{w})$ such that $p \cap \mathbb{O}=r \cap \mathbb{O}=\varnothing$ has precisely one such decomposition and exactly one other decomposition as $r^{\prime} * p^{\prime}$, where $r_{\tilde{\phi}}^{\prime} \in \operatorname{Rect}_{G_{\beta}}^{o}\left(\mathbf{x}, \mathbf{y}^{\prime}\right)$ and $p^{\prime} \in \operatorname{Pent}_{\beta \gamma}^{o}\left(\mathbf{y}^{\prime}, \mathbf{w}\right)$ and $r^{\prime} \cap \mathbb{O}=p^{\prime} \cap \mathbb{O}=\varnothing$. It follows that $\tilde{\phi}$ is a chain map and, so, induces a map

$$
\widetilde{\Phi}: \widetilde{H F L}\left(m\left(T_{w \sigma_{i}}\right)\right) \rightarrow \widetilde{H F L}\left(m\left(T_{w}\right)\right) \text {. }
$$

Moreover, it is clear that $\operatorname{Pent}_{\beta \gamma}^{o}\left(\mathbf{z}^{+}\left(G_{\beta}\right), \mathbf{z}^{+}\left(G_{\gamma}\right)\right)$ consists only of the shaded pentagon shown in Figure 4, and that $\operatorname{Pent}_{\beta \gamma}^{o}\left(\mathbf{z}^{+}\left(G_{\beta}\right), \mathbf{y}\right)$ is empty for $\mathbf{y} \neq \mathbf{z}^{+}\left(G_{\gamma}\right)$. Therefore, $\widetilde{\Phi}$ sends $\tilde{\theta}\left(T_{w \sigma_{i}}\right)$ to $\tilde{\theta}\left(T_{w}\right)$, proving Theorem 1.1.

The more general comultiplication fact stated in Theorem 1.2 follows from the above result together with the sequence of braid moves depicted in Figure 5: The braid in (a) represents the transverse link $T_{h g}$. The braid in (b) is obtained from that in (a) by a mixture of isotopy and positive stabilizations. The braid in (c) is obtained from that in (b) by isotopy followed by the introduction of negative crossings. The braid in (e) is isotopic to the braids in (c) and (d), and represents the connected sum of the transverse links $T_{g}$ and $T_{h}$ (for the latter statement, see Birman and Menasco [4]). Therefore, a composition of the maps $\widetilde{\Phi}$ described above (one for each negative crossing introduced in going from (b) to (c)) yields a map

$$
\tilde{\mu}: \widetilde{H F L}\left(m\left(T_{h g}\right)\right) \rightarrow \widetilde{H F L}\left(m\left(T_{g} \# T_{h}\right)\right)
$$

which sends $\tilde{\theta}\left(T_{h g}\right)$ to $\tilde{\theta}\left(T_{g} \# T_{h}\right)$.

(a)

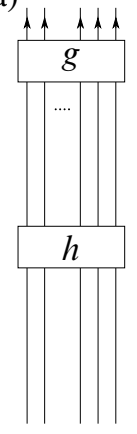

(b)

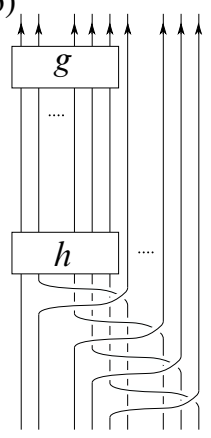

(c)

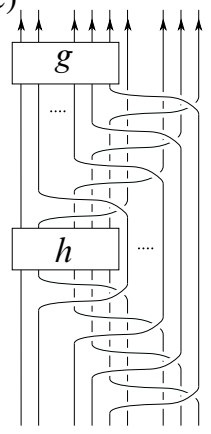

(d)

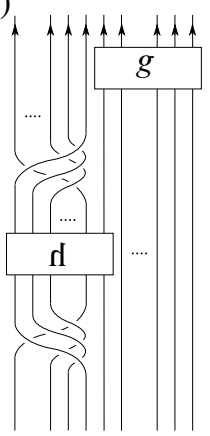

(e)

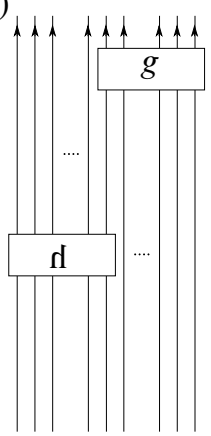

Figure 5: $T_{h g}$ is transversely isotopic to the braid in (b), which, after introducing negative crossings (or, equivalently, up to braid isotopy, getting rid of positive crossings) is transversely isotopic to $T_{g} \# T_{h}$, which is represented by the braid in (e).

Suppose that $T_{g} \# T_{h}$ is any connected sum of $T_{g}$ and $T_{h}$. In [28], Vértesi proves the following refinement of the Künneth formula described in [24, Theorem 1.4]. (Her 
proof is actually for the analogous result in knot Floer homology, but it extends in an obvious manner to a proof of the theorem below.)

Theorem 4.2 There is an isomorphism

$$
\widehat{H F L}\left(m\left(T_{g} \# T_{h}\right)\right) \cong \widehat{H F L}\left(m\left(T_{g}\right)\right) \otimes_{\mathbb{Z}_{2}} \widehat{H F L}\left(m\left(T_{h}\right)\right)
$$

under which $\hat{\theta}\left(T_{g} \# T_{h}\right)$ is identified with $\hat{\theta}\left(T_{g}\right) \otimes \hat{\theta}\left(T_{h}\right)$.

Vértesi's theorem, used in combination with the comultiplication map $\tilde{\mu}$, may be applied to prove Theorem 1.3.

Proof of Theorem 1.3 Recall from the previous section that $\hat{\theta}\left(T_{w}\right)$ is nonzero if and only if $\tilde{\theta}\left(T_{w}\right)$ is nonzero. If $\hat{\theta}\left(T_{g}\right)$ and $\hat{\theta}\left(T_{h}\right)$ are both nonzero, then, by Theorem 4.2, so is $\hat{\theta}\left(T_{g} \# T_{h}\right)$, and, hence, so is $\tilde{\theta}\left(T_{g} \# T_{h}\right)$. Since $\tilde{\mu}$ sends $\tilde{\theta}\left(T_{h g}\right)$ to $\tilde{\theta}\left(T_{g} \# T_{h}\right)$ this implies that $\tilde{\theta}\left(T_{h g}\right)$ is nonzero, and, hence, so is $\hat{\theta}\left(T_{h g}\right)$.

Recall that a braid $T_{g}$ is said to be quasipositive if $g \in B_{n}$ can be expressed as a product of conjugates of the form $w \sigma_{i} w^{-1}$, where $w$ is any word in $B_{n}$.

Corollary 4.3 If $T_{g}$ is a quasipositive braid, then $\hat{\theta}\left(T_{g}\right) \neq 0$.

Proof of Corollary 4.3 If $g$ is a product of $m$ conjugates as above, then after resolving the corresponding $m$ positive crossings, one obtains a braid isotopic to $I_{n}$, the trivial $n-$ braid. Therefore, a composition of $m$ of the maps $\widetilde{\Phi}$ sends $\tilde{\theta}\left(T_{g}\right)$ to $\tilde{\theta}\left(I_{n}\right)$. Moreover, one sees by glancing at the grid diagram for $I_{n}$ in Figure 6 that $\tilde{\theta}\left(T_{I_{n}}\right) \neq 0$. Therefore, $\tilde{\theta}\left(T_{g}\right) \neq 0$ and the same is true of $\hat{\theta}\left(T_{g}\right)$.

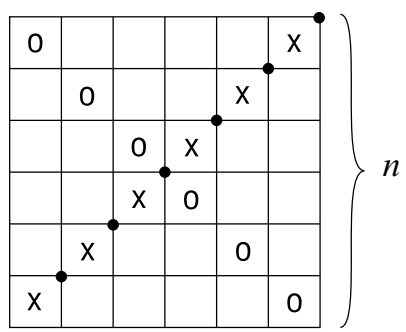

Figure 6: A grid diagram $G_{n}$ for the trivial braid $I_{n}$. It is straightforward to check that the cycle $\mathbf{z}^{+}\left(G_{n}\right) \in \mathbf{S}\left(G_{n}\right)$, represented by the collection of black dots, is not a boundary in $\widetilde{C F L}\left(m\left(I_{n}\right)\right)$. 
Proof of Theorem 1.4 Suppose that $T_{w}$ is a positive braid with one component. Then $\hat{\theta}\left(T_{w}\right) \neq 0$, by the corollary above; also, $T_{w}$ is a fibered knot [6]. Moreover, $\hat{\theta}\left(T_{w}\right)$ lies in Alexander grading $\left(\operatorname{sl}\left(T_{w}\right)+1\right) / 2$, which, in this case, is simply the genus of $T_{w}$ [25]. Therefore, $\widehat{\theta}\left(T_{w}\right)$ is the unique generator of $\widehat{H F L}\left(T_{w}, g\left(T_{w}\right)\right)$. To show that $\hat{\theta}\left(T_{w}\right)=\underline{\hat{\theta}}\left(T_{w}\right)$, it suffices to prove that $\underline{\hat{\theta}}\left(T_{w}\right)$ is nonzero as well. Fortunately, this has been shown by Vela-Vick in [27].

\section{Finding new transversely nonsimple links}

In this section, we outline and apply one strategy for using comultiplication (in particular, Theorem 1.3) to generate a plethora of new examples of transversely nonsimple link types. Consider the braid words

$$
w_{1}=a \sigma_{1}^{m} b \sigma_{1}^{-1} c \quad \text { and } \quad w_{2}=a \sigma_{1}^{-1} b \sigma_{1}^{m} c
$$

in $B_{n}$, where $a, b$ and $c$ are words in the generators $\sigma_{2}, \ldots, \sigma_{n-1}$. The transverse braids $T_{w_{1}}$ and $T_{w_{2}}$ are said to be related by a negative flype and, in particular, represent the same topological link type. If, in addition, $m$ is odd, or if $m$ is even and the two strands which cross according to $\sigma_{1}^{m}$ belong to the same component of $T_{w_{1}}$, then $\mathcal{S L}\left(T_{w_{1}}\right)=\mathcal{S} \mathcal{L}\left(T_{w_{2}}\right)$.

Suppose that $\hat{\theta}\left(T_{w_{1}}\right)=0$ and $\hat{\theta}\left(T_{w_{2}}\right) \neq 0$. The idea is to find a word $h$ in the generators $\sigma_{2}, \ldots, \sigma_{n-1}$ with $\hat{\theta}\left(T_{h}\right) \neq 0$. Theorem 1.3 would then imply that $\hat{\theta}\left(T_{h w_{2}}\right) \neq 0$. If it is also true that $\hat{\theta}\left(T_{h w_{1}}\right)=0$, then $T_{h w_{1}}$ and $T_{h w_{2}}$ are not transversely isotopic although they are topologically isotopic. We would like to find examples which also satisfy $\mathcal{S} \mathcal{L}\left(T_{h w_{1}}\right)=\mathcal{S} \mathcal{L}\left(T_{h w_{2}}\right)$ (if $T_{h w_{1}}$ is a knot, this is automatic) so as to produce topological link types which are not transversely simple. One nice feature of this proposed method, which differs from that in [28], is that there is no reason to believe $a$ priori that the link $T_{h w_{1}}$ so obtained is composite.

In principle, Theorem 1.3 eliminates half of the work in this scenario - namely, showing that $\hat{\theta}\left(T_{h w_{2}}\right) \neq 0$. In practice, one would like to find examples in which the other half - showing that $\hat{\theta}\left(T_{h w_{1}}\right)$ is zero - is very easy. To that end, one strategy is to pick an example in which $T_{w_{1}}$ is transversely isotopic to a braid which can be negatively destabilized, and to show that the same is true of the braid $T_{h w_{1}}$, which would guarantee that $\hat{\theta}\left(T_{h w_{1}}\right)=0$ by Corollary 3.3. In particular, $T_{w_{1}}$ must belong to a topological link type with a transverse representative (that is, $T_{w_{2}}$ ) which does not maximize self-linking number, but which cannot be negatively destabilized.

The most well-known such link type is that of the $(2,3)$ cable of the $(2,3)$ torus knot. In [12], Etnyre and Honda prove the following. 
Proposition 5.1 The $(2,3)$ cable of the $(2,3)$ torus knot has two Legendrian representatives, $L_{1}$ and $L_{2}$, both with $t b=5$ and $r=2$, for which $L_{1}$ is the positive (Legendrian) stabilization of a Legendrian knot while $L_{2}$ is not. Moreover, $L_{1}$ and $L_{2}$ are not Legendrian isotopic after any number of negative (Legendrian) stabilizations.

That $L_{1}$ and $L_{2}$ are not Legendrian isotopic after any number of negative stabilizations implies that their transverse pushoffs, $L_{1}^{+}$and $L_{2}^{+}$, are not transversely isotopic (yet, they both have $s l=3$ ). Moreover, since $L_{1}$ is the positive stabilization of a Legendrian knot, its pushoff $L_{1}^{+}$is transversely isotopic to the negative stabilization of some transverse braid.

Matsuda and Menasco have since given explicit forms for $L_{1}$ and $L_{2}$ [18]. Figures 7 (a) and $7\left(\mathrm{a}^{\prime}\right)$ depict the rectangular diagrams corresponding to slightly modified versions of these forms (ours are derived from the front diagrams in [20, Figure 6]). The

(a)

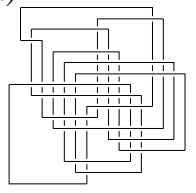

$\left(a^{\prime}\right)$

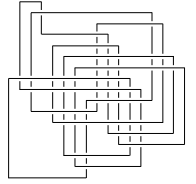

(b)

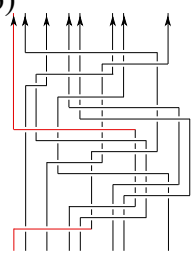

(c)

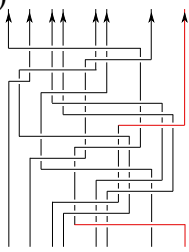

$\left(c^{\prime}\right)$
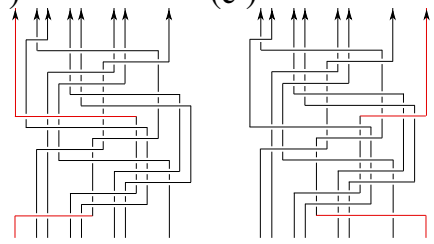

(d)

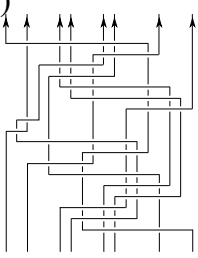

$\left(d^{\prime}\right)$

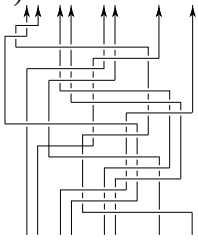

(e)

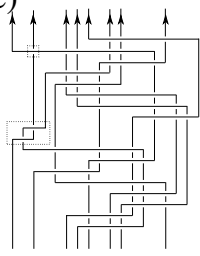

$\left(\mathrm{e}^{\prime}\right)$

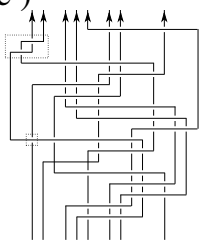

Figure 7: On the top, the rectangular diagrams for $L_{1}$ and its transverse pushoff $L_{1}^{+}$. On the bottom, those for $L_{2}$ and $L_{2}^{+}$. The circled regions in (e) and (e') indicate that $L_{1}^{+}$and $L_{2}^{+}$are transversely isotopic to braids related by a negative flype.

rectangular braid diagrams for the transverse pushoffs $L_{1}^{+}$and $L_{2}^{+}$are shown in (b) and $\left(b^{\prime}\right)$, respectively. The braids in (c) and $\left(c^{\prime}\right)$ are obtained from those in (b) and $\left(b^{\prime}\right)$ by isotoping the red arcs as indicated, and the braids in (d) and $\left(\mathrm{d}^{\prime}\right)$ are obtained from those in (c) and ( $\left.\mathrm{c}^{\prime}\right)$ after additional simple isotopies and conjugations. The braids in (e) and $\left(\mathrm{e}^{\prime}\right)$ are obtained from those in $(\mathrm{d})$ and $\left(\mathrm{d}^{\prime}\right)$ by conjugation, and they are related to one another by a negative flype. Indeed, Figure 7 shows that $L_{1}^{+}$and $L_{2}^{+}$ are transversely isotopic to the transverse braids $T_{w_{1}}$ and $T_{w_{2}}$, respectively, where 


$$
\begin{aligned}
w_{1} & =a \sigma_{1}^{2} b \sigma_{1}^{-1} c, w_{2}=a \sigma_{1}^{-1} b \sigma_{1}^{2} c, \\
a & =\sigma_{4} \sigma_{3} \sigma_{5} \sigma_{6} \sigma_{4} \sigma_{5} \sigma_{5} \sigma_{6} \sigma_{4} \sigma_{5} \sigma_{7} \sigma_{6} \sigma_{5}^{-1} \sigma_{4}^{-1} \sigma_{3}^{-1} \sigma_{2} \sigma_{3} \sigma_{3} \sigma_{4} \sigma_{5} \sigma_{4}^{-1} \sigma_{3}^{-1} \sigma_{2}^{-1}, \\
b & =\sigma_{5} \sigma_{6} \sigma_{7} \sigma_{6}^{-1} \sigma_{5}^{-1} \sigma_{4}^{-1} \sigma_{6}^{-1} \sigma_{5}^{-1} \sigma_{4}^{-1} \sigma_{3} \sigma_{4} \sigma_{5} \sigma_{2} \sigma_{3} \sigma_{4} \sigma_{4} \sigma_{5} \sigma_{6} \sigma_{5}^{-1} \sigma_{4}^{-1} \sigma_{3}^{-1} \sigma_{2}^{-1}, \\
c & =\sigma_{7}^{-1} \sigma_{6}^{-1} \sigma_{5}^{-1} .
\end{aligned}
$$

According to Proposition 5.1, $T_{w_{1}}$ is transversely isotopic to a braid which can be negatively destabilized. Figure 8 shows a sequence of transverse braid moves which demonstrates that the same is true of $T_{h w_{1}}$ for any word $h \in B_{8}$ in the generators $\sigma_{3}, \ldots, \sigma_{6}$. The braid in (b) is obtained from that in (a) by isotoping the red and blue

(a)

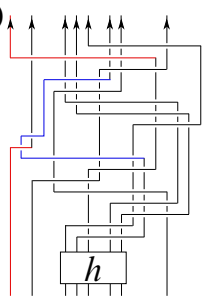

(f)

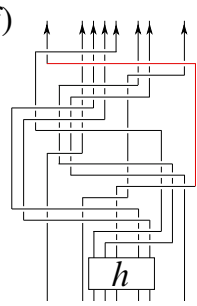

(b)

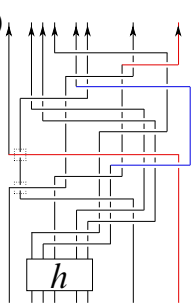

(g)

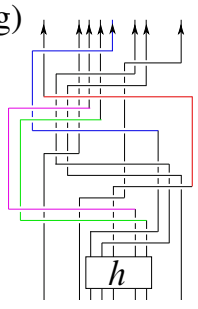

(c)

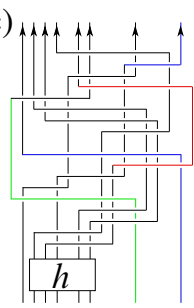

(h)

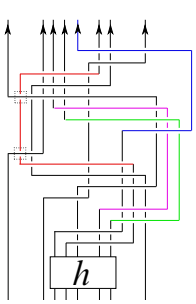

(d)

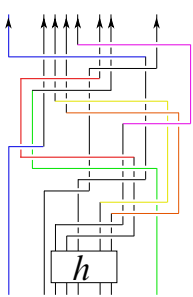

(i)

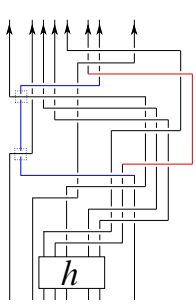

(e)

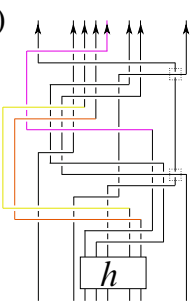

(j)

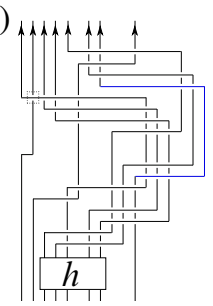

Figure 8: (a) shows a rectangular braid diagram for $T_{h w_{1}}$. After a sequence of braid isotopies and exchange moves, we obtain the braid in (j), which can be negatively destabilized.

arcs as shown. The braid in (c) is related to that in (b) by an exchange move at the circled crossings in (b). The braid in (d) is obtained from that in (c) by isotopy of the red, blue and green arcs. The braid in (e) is obtained from that in (d) after the indicated isotopy of the yellow, orange and purple arcs. An exchange move at the circled crossings in (e) produces the braid in (f). The braid in (g) is obtained from that in (f) by isotoping the red arc as shown. The braid in (h) is obtained from that in (g) after an isotopy of the blue, green and purple arcs as shown. An exchange move at the circled crossings in (h), followed by the indicated isotopy of the red arc produces the braid in (i). Finally, the braid in (j) is obtained from that in (i) by an exchange move at the circled crossings in (i), followed by the indicated isotopy of the blue arc. 
Note that the braid in (j) may be negatively destabilized at the circled crossing. The essential point here is that the region of the braid in (a) corresponding to the word $h$ is not affected by this combination of isotopies and exchange moves.

To sum up: since $\hat{\theta}\left(T_{w_{2}}\right) \neq 0$ (see Ng, Ozsváth and Thurston [20]), we have proven that for any $h \in B_{8}$ which is (1) a word in the generators $\sigma_{3}, \ldots, \sigma_{6}$ and for which (2) $\hat{\theta}\left(T_{h}\right) \neq 0$, it is the case that $\hat{\theta}\left(T_{h w_{1}}\right)=0$ while $\hat{\theta}\left(T_{h w_{2}}\right) \neq 0$. It follows that the transverse braids $T_{h w_{1}}$ and $T_{h w_{2}}$ are not transversely isotopic though they are topologically isotopic. If, in addition, (3) $h$ is such that the two strands of $T_{h w_{1}}$ which cross according to the string $\sigma_{1}^{2}$ belong to the same component of $T_{h w_{1}}$, then $\mathcal{S} \mathcal{L}\left(T_{h w_{1}}\right)=\mathcal{S} \mathcal{L}\left(T_{h w_{2}}\right)$; that is, the topological link type represented by $T_{h w_{1}}$ is transversely nonsimple.

There are infinitely many choices of $h$ which meet criteria (1)-(3) above. In order to give such an $h$, we first prove the following.

Lemma 5.2 For $1 \leq j \leq k$ and $0 \leq l \leq k-j$, consider the map $\psi_{j, k, l}: B_{j} \rightarrow B_{k}$ which sends $\sigma_{i}$ to $\sigma_{i+l}$. If $g$ is a word in $B_{j}$ for which $\hat{\theta}\left(T_{g}\right) \neq 0$, and $h=\psi_{j, k, l}(g)$, then $\hat{\theta}\left(T_{h}\right) \neq 0$ as well.

See Figure 9 for a pictorial depiction of the map $\psi_{j, k, l}$.

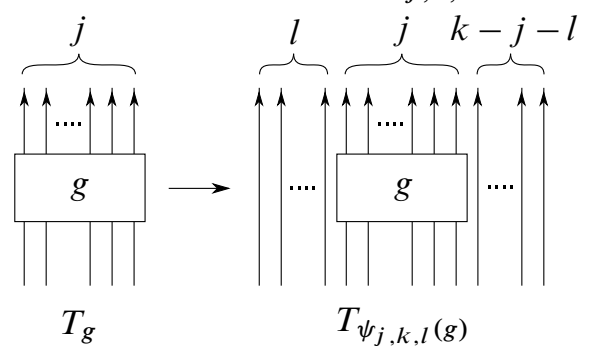

Figure 9: On the left, the $j$-braid $T_{g}$. On the right, the $k$-braid $T_{\psi_{j, k, l}(g)}$.

Proof of Lemma 5.2 If $h=\psi_{j, k, l}(g)$, then the braid $T_{h}$ is easily seen to be connected sum of $T_{g}$ with the trivial braids $I_{k-j-l+1}$ and $I_{l+1}$. We know, from the proof of Corollary 4.3 , that $\hat{\theta}\left(I_{n}\right) \neq 0$ for any $n \geq 1$. Lemma 5.2 therefore follows from Theorem 4.2.

It follows from Corollary 4.3 and from Lemma 5.2 that $h=\psi_{4,8,3}(g)$ satisfies criteria (1) and (2) above as long as $T_{g}$ is a quasipositive 4-braid. Let $h=\psi_{4,8,3}(g)$ for

$$
g=\sigma_{3} \sigma_{2} \sigma_{3} \sigma_{1} \sigma_{2} \sigma_{3}
$$

It is easy to check that $h^{n}$ also satisfies criterion (3) (as well as criteria (1) and (2), of course) for all $n \geq 0$. 
Corollary 5.3 The topological link types represented by $T_{h^{n} w_{1}}$ are transversely nonsimple for all $n \geq 0$. When $n$ is even, $T_{h^{n} w_{1}}$ is a knot; otherwise, $T_{h^{n} w_{1}}$ is a 3-component link.

Below, we prove that most of the links in Corollary 5.3 are prime. Note that $T_{h^{n} w_{1}}$ is obtained from $T_{w_{1}}$ by performing $n$ positive half twists of strands 4-7 in the region of $T_{w_{1}}$ where we would insert the word $h^{n}$. For $n=2 m$, this amounts to adding $m$ positive full twists, which can also be accomplished by performing $-1 / m$ surgery on an unknot $U$ encircling strands 4-7 of $T_{w_{1}}$ in the corresponding region. See Figure 10.
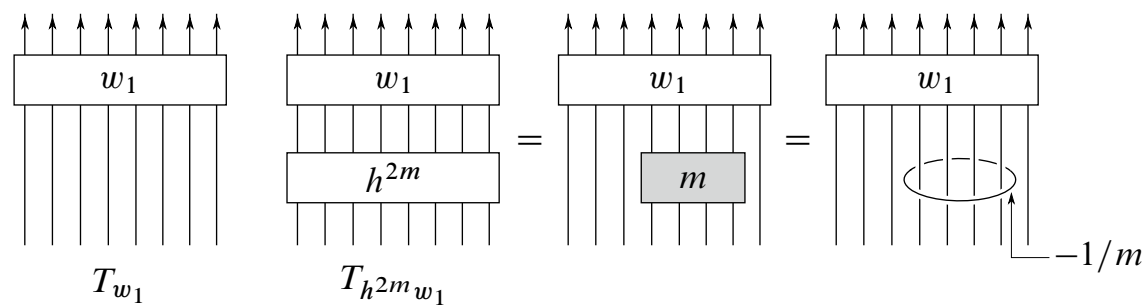

Figure 10: $T_{h^{2 m} w_{1}}$ is obtained from $T_{w_{1}}$ by performing $m$ positive full twists of strands $4-7$, or, equivalently, by performing $-1 / m$ surgery on the unknot shown on the right.

A SnapPea computation [29] combined with the Inverse Function Theorem test described in Moser's thesis [19] shows that the complement of the link $T_{w_{1}} \cup U$ is hyperbolic. To be specific, SnapPea finds a triangulation of this link complement by ideal tetrahedra and computes an approximate solution to the gluing equations. Moser's test then confirms, using this approximate solution, that an exact solution exists.

Thurston's celebrated Dehn Surgery Theorem then implies that all but finitely many Dehn fillings of the boundary component corresponding to $U$ are hyperbolic as well [26]. In turn, this implies that the link $T_{h^{2 m}} w_{1}$ is hyperbolic, and, hence, prime for all but finitely many $m$. This argument can be repeated to show that the links $T_{h^{2 m+1} w_{1}}$ are also prime for all but finitely many $m$. The lemma below sums this up.

Lemma 5.4 The links $T_{h^{n} w_{1}}$ are prime for all but finitely many values of $n$.

\section{References}

[1] K Baker, J B Etnyre, J Van Horn-Morris, Fibered transverse knots and the Bennequin bound arXiv:0803.0758 
[2] J A Baldwin, Comultiplicativity of the Ozsváth-Szabó contact invariant, Math. Res. Lett. 15 (2008) 273-287 MR2385640

[3] D Bennequin, Entrelacements et équations de Pfaff, from: “Third Schnepfenried geometry conference, Vol. 1 (Schnepfenried, 1982)", Astérisque 107, Soc. Math. France, Paris (1983) 87-161 MR753131

[4] J S Birman, W W Menasco, Studying links via closed braids. IV. Composite links and split links, Invent. Math. 102 (1990) 115-139 MR1069243

[5] J S Birman, W W Menasco, Stabilization in the braid groups. II. Transversal simplicity of knots, Geom. Topol. 10 (2006) 1425-1452 MR2255503

[6] J S Birman, R F Williams, Knotted periodic orbits in dynamical systems. I. Lorenz's equations, Topology 22 (1983) 47-82 MR682059

[7] Y Eliashberg, Legendrian and transversal knots in tight contact 3-manifolds, from: "Topological methods in modern mathematics (Stony Brook, NY, 1991)", (L R Goldberg, A V Phillips, editors), Publish or Perish, Houston (1993) 171-193 MR1215964

[8] J Epstein, D Fuchs, M Meyer, Chekanov-Eliashberg invariants and transverse approximations of Legendrian knots, Pacific J. Math. 201 (2001) 89-106 MR1867893

[9] J B Etnyre, Transversal torus knots, Geom. Topol. 3 (1999) 253-268 MR1714912

[10] J B Etnyre, K Honda, Knots and contact geometry. I. Torus knots and the figure eight knot, J. Symplectic Geom. 1 (2001) 63-120 MR1959579

[11] J B Etnyre, K Honda, On connected sums and Legendrian knots, Adv. Math. 179 (2003) 59-74 MR2004728

[12] J B Etnyre, K Honda, Cabling and transverse simplicity, Ann. of Math. (2) 162 (2005) 1305-1333 MR2179731

[13] K Kawamuro, Connect sum and transversely nonsimple knots, Math. Proc. Cambridge Philos. Soc. 146 (2009) 661-669 MR2496350

[14] T Khandhawit, L Ng, A family of transversely nonsimple knots, Algebr. Geom. Topol. 10 (2010) 293-314

[15] P Lisca, P Ozsváth, A Stipsicz, Z Szabó, Heegaard Floer invariants of Legendrian knots in contact three-manifolds arXiv:0802.0628

[16] C Manolescu, P Ozsváth, S Sarkar, A combinatorial description of knot Floer homology, Ann. of Math. (2) 169 (2009) 633-660 MR2480614

[17] C Manolescu, P Ozsváth, Z Szabó, D Thurston, On combinatorial link Floer homology, Geom. Topol. 11 (2007) 2339-2412 MR2372850

[18] W W Menasco, H Matsuda, An addendum on iterated torus knots (appendix) arXiv: math.GT/0610566 
[19] H H Moser, Proving a manifold to be hyperbolic once it has been approximated to be so, ProQuest LLC, Ann Arbor, MI (2005) MR2621880 PhD Thesis-Columbia University

[20] L Ng, P Ozsváth, D Thurston, Transverse knots distinguished by knot Floer homology, J. Symplectic Geom. 6 (2008) 461-490 MR2471100

[21] L Ng, D Thurston, Grid diagrams, braids, and contact geometry, from: "Proceedings of Gökova Geometry-Topology Conference 2008”, (S Akbulut, T Önder, R J Stern, editors) (2009) 120-136 MR2500576

[22] S Y Orevkov, V V Shevchishin, Markov theorem for transversal links, J. Knot Theory Ramifications 12 (2003) 905-913 MR2017961

[23] P Ozsváth, A Stipsicz, Contact surgeries and the transverse invariant in knot Floer homology arXiv:0803.1252

[24] P Ozsváth, Z Szabó, Holomorphic disks, link invariants and the multi-variable Alexander polynomial, Algebr. Geom. Topol. 8 (2008) 615-692 MR2443092

[25] P Ozsváth, Z Szabó, D Thurston, Legendrian knots, transverse knots and combinatorial Floer homology, Geom. Topol. 12 (2008) 941-980 MR2403802

[26] W P Thurston, The geometry and topology of three-manifolds, Princeton Univ. Math. Dept. Lecture Notes (1979) Available at http://msri.org/publications/books/ gt $3 \mathrm{~m} /$

[27] DS Vela-Vick, On the transverse invariant for bindings of open books arXiv: 0806.1729

[28] V Vértesi, Transversely nonsimple knots, Algebr. Geom. Topol. 8 (2008) 1481-1498 MR2443251

[29] J Weeks, SnapPea Available at http://www.geometrygames.org/SnapPea

[30] N Wrinkle, The Markov theorem for transverse knots arXiv:math.GT/0202055

Department of Mathematics, Princeton University

Princeton, NJ 08544-1000

baldwinj@math.princeton.edu

http://www.math.princeton.edu/ baldwinj

Received: 19 October 2009 Revised: 16 February 2010 